\title{
Úber Proteine und deren Abbauprodukte, XIX* STERISCHE UND INDUKTIVE EFFEKTE BEI DER HYDROLYSE VON POLYPEPTIDEN
}

\author{
von Kurt Heyns und Hans Friedrich Grützmacher
}

Aus dem Chemischen Staatsinstitut der Universität Hamburg, Institut für Organische Chemie Eingegangen am 2. Dezember 1960

\begin{abstract}
Die Hydrolyse von Poly-DL-[p-methoxy-phenyl]-alanin, Poly-DL-phenylalanin und Poly-DL-[p-nitro-phenyl]-alanin in einer Mischung von Dichloressigsäure und wäßrigem $\mathrm{HCl}$ (DCE-HCl) bei $37^{\circ}$ wurde untersucht. Weder die Spaltung einer Endbindung noch die Spaltung einer inneren Bindung der Peptidkette wird durch induktive Effekte der Aminosäureseitenkette beeinflußt. - Dialanyl${ }^{15} \mathrm{~N}$-alanin wurde dargestellt und dessen Hydrolyse bei $37^{\circ}$ massenspektrometrisch verfolgt. In wäßrigem $\mathrm{HCl}$ wird dieses Tripeptid an der $\mathrm{C}$-endständigen Bindung gespalten, in $\mathrm{DCE}-\mathrm{HCl}$ an der $\mathrm{N}$-endständigen Bindung. Die Aktivierungsenergien und Aktivierungsentropien der sauren Hydrolyse von Poly-DL-alanin, Poly-DL- $\alpha$-amino-buttersäure, Poly-DL-norleucin, Poly-DLphenylalanin, Poly-DL-leucin und Poly-DL-isoleucin wurden ermittelt. Die Ergebnisse lassen sich durch sterische Einflüsse der Aminosäureseitenketten hinreichend erklären.
\end{abstract}

Es wurde versucht, über den Zusammenhang der Struktur von Aminosäuren und der Stabilität der von ihnen gebildeten Peptidbindungen gegenüber der sauren Hydrolyse weiteren Aufschluß zu bekommen.

Durch Messung der Hydrolysengeschwindigkeit in DCE- $\mathrm{HCl}$ bei $37^{\circ} 1,2$ ) wurden für die Stabilität der Peptidbindungen folgende Reihen gefunden: Spaltung einer endständigen Peptidbindung (RG-Konstante $\mathrm{k}_{\mathrm{E}}$ ): Polyglycin < Poly-DL-alanin < Poly-DL- $\alpha$-amino-buttersäure $\cong$ Poly-DL-phenylalanin < Poly-DL-norleucin < Poly-DL-leucin < Poly-DL-isoleucin. Spaltung einer inneren Peptidbindung (RG-Konstante $\mathrm{k}_{1}$ ): Polyglycin < Poly-DL-alanin < PolyDL- $\alpha$-amino-buttersäure $\cong$ Poly-DL-norleucin $\cong$ Poly-DL-leucin $\cong$ Poly-DL-phenylalanin $<$ Poly-DL-isoleucin. - Es wurde angenommen, daß die Stabilität einer inneren Peptidbindung vorwiegend durch sterische Effekte bewirkt wird, während an einer Endbindung auch induktive Effekte wirksam sind. Dafür sprach die Stellung des Phenylalanins in beiden Reihen: die dem Isobutylrest vergleichbare Raumerfüllung der Benzylgruppe weist ihm einen Platz an der Seite des Leucins an $\left(k_{I}\right)$, während der - I-Effekt des Benzylrestes zu einer Verminderung der Stabilität führt $\left(\mathrm{k}_{\mathrm{E}}\right)$.

*) XVIII. Mitteilung: K. HeYns und G. Legler, Hoppe-Seyler's Z. physiol Chem. 321, 184 (1960).

1) K. Heyns, W. Walter und H. F. Grützmacher, Liebigs Ann. Chem. 609, 209 (1957).

2) K. Heyns, H. F. Grützmacher und W. Walter, Liebigs Ann. Chem. 609, 224 (1957). 
Vor allem sollte jetzt überprüft werden, ob an den Endbindungen tatsächlich induktive Effekte auftreten. Dazu wurden folgende Versuchsreihen durchgeführt:

A. Hydrolyse von Polypeptiden, bei denen die sterischen Einflüsse weitgehend konstant gehalten werden, die induktiven Effekte jedoch variieren. Für diesen Zweck bieten sich substituierte aromatische Aminosäuren an. Gemessen wurde die Hydrolyse von Poly-DL-phenylalanin, Poly-DL-[ $p$-methoxy-phenyl]-alanin und Poly-DL-[p-nitrophenyl]-alanin bei $37^{\circ}$.

B. Bestimmung der Hydrolysengeschwindigkeit der C- und N-endständigen Peptidbindung. Mit Ausnahme von Polyglycin werden die untersuchten Polyaminosäuren vorwiegend vom Kettenende her abgebaut, dabei wird $\mathbf{k}_{\mathrm{E}}$ des Poly-DL-alanins beim Ưbergang vom Wasser zu DCE als Lösungsmittel wesentlich stärker erhöht als $\mathbf{k}_{\mathbf{I}}$. Zwischen einem Abbau vom Amino- und Carboxylende her konnte mit den verwendeten Methoden nicht unterschieden werden. Wir haben daher ein Dialanylalanin synthetisiert, dessen $\mathrm{C}$-endständiges Alanin mit ${ }^{15} \mathrm{~N}$ markiert war, und haben dessen Hydrolyse in wäßrigem $\mathrm{HCl}$ und in $\mathrm{DCE}-\mathrm{HCl}$ verfolgt.

C. Bestimmung der Aktivierungsenergie und Aktivierungsentropie. Dazu wurde die Hydrolyse der Polyaminosäuren bei $25^{\circ}, 37^{\circ}, 45^{\circ}$ und $54.4^{\circ}$ gemessen.

\section{METHODIK UND KINETTK}

Die Polyaminosäuren wurden durch Polymerisation der entsprechenden Oxazoliddione-(2.5) in Dioxan mit Wasser als Polymerisationsstarter hergestellt. Der mittlere Polymerisationsgrad lag zwischen 10 und 55. Einzelheiten des Hydrolysenverfahrens ${ }^{2)}$ : Bestimmt wurden die entstehenden freien Aminosäuren nach KEMBLE und MACPHERSON ${ }^{3)}$ und die freiwerdenden Aminogruppen nach VAN SLYKE in einer Mikroapparatur4). Die Werte für die Aminosäurekonzentration und die der Peptidkonzentration, die sich aus den Differenzen der Konzentration der freien Aminogruppen und der Aminosäurekonzentration ergeben, wurden gegen die Hydrolysenzeit aufgetragen und die RG-Konstanten graphisch ermittelt. Diese Methode zur Bestimmung der Anfangsgeschwindigkeit ist nicht sehr genau; der Fehler der einzelnen RG-Konstanten kann $\pm 20 \%$ betragen.

\section{A. Hydrolyse der Polyphenylalanine}

Die Ergebnisse der Versuche mit Poly-DL-[p-methoxy-phenyl]-alanin, Poly-DLphenylalanin und Poly-DL-[p-nitro-phenyl]-alanin in DCE-HCl (ca. 65 Vol.-\% DCE; $3.6-4.3 n \mathrm{HCl}$ ) sind in Tabelle 1 zusammengefaßt. Um die einzelnen Hydrolysenkonstanten vergleichen zu können, wurden sie durch die jeweilige analytische $\mathrm{HCl}$ Konzentration dividiert.

3) A. R. Kemble und H. T. MAcPherson, Biochem. J. 56, 548 (1954).

4) G. KaINZ, Mikrochim. Acta 1953, 349. 
Tabelle 1. Hydrolysenkonstanten der Poly-DL-phenylalanine bei $37^{\circ}$ in Sek.-1

$\begin{array}{lcc}\text { Poly-DL-[p-methoxy-phenyl]-alanin } & \mathrm{k}_{\mathrm{E}}=23.0 \cdot 10^{-8} & \mathrm{k}_{\mathrm{I}}=6.1 \cdot 10^{-8} \\ \text { Poly-DL-phenylalanin } & 21.3 & 7.6 \\ \text { Poly-DL-[p-nitro-phenyl]-alanin } & 11.7 & 8.0\end{array}$

Mit Ausnahme von $\mathrm{k}_{\mathrm{E}}$ des Poly-DL-[p-nitro-phenyl]-alanins sind $\mathrm{k}_{\mathrm{E}}$ und $\mathrm{k}_{\mathrm{I}}$ der drei Polyaminosäuren praktisch gleich. Im Gegensatz $\mathrm{zu}$ der ursprünglich gemachten Annahme tritt also auch bei der Hydrolyse einer Endbindung kein induktiver Effekt auf.

Den tiefen $\mathbf{k}_{\mathrm{E}}$-Wert des Poly-DL-[p-nitro-phenyl]-alanins kann man nicht erklären durch den -I-Effekt der $p$-Nitro-benzyl-Seitenkette, da bei einer direkten Wirkung die Hydrolysengeschwindigkeit erhöht werden müßte. Nimmt man an, daB der Spaltung der Peptidbindung die Anlagerung eines Protons an die - $\mathrm{CO}-\mathrm{NH}$-Gruppe vorausgeht und daß die $p$-Nitrobenzyl-Gruppe dieses Gleichgewicht zu Ungunsten der protonierten Peptidbindung verschiebt, so sollte nicht nur die Endbindung, sondern auch eine innere Bindung langsamer gespalten werden, was aber nicht beobachtet wurde. Ein Wechsel vom $A_{2}$ - zum $A_{1}$-Mechanismus, wie ihn MarTiN5) bei der Hydrolyse in reiner Essigsäure fand, scheidet wegen des relativ großen Wassergehaltes des Hydrolysiergemisches aus. Möglicherweise kommt in dem kleinen $\mathbf{k}_{\mathrm{E}}$ des Poly-DL-[p-nitro-phenyl]-alanins die herabgesetzte Basizität der Aminogruppe des Nitrophenylalanins ( $\mathrm{p} K \sim 8.4$ gegenüber 9.7 der anderen Aminosäuren) zum Ausdruck. Die Bedeutung der $\mathrm{NH}_{3}{ }^{\oplus}$-Endgruppe der Peptide bei der Hydrolyse in DCE-Mischungen zeigte sich in der Versuchsreihe B.

Von den früher ${ }^{2)}$ für Poly-DL-phenylalanin angegebenen $k$-Werten ist $k_{E}=$ $49.7 \cdot 10^{-8}$ Sek. $^{-1}$ zu hoch.

$\mathrm{Zu}$ große $\mathrm{k}_{\mathrm{E}}$-Werte entstehen auf folgende Weise: Wir haben den Polymerisationsgrad der Polyaminosäuren durch Bestimmung der Endaminogruppen nach vaN SLYKE ermittelt und zur Kontrolle auch die $\mathrm{CO}_{2} \mathrm{H}$-Endgruppen durch Titration mit $\mathrm{CH}_{3} \mathrm{ONa}$ in Dioxan oder Dimethylformamid bestimmt ${ }^{6}$. Man erfaßt jedoch gar nicht die $\mathrm{CO}_{2} \mathrm{H}$-Endgruppen, sondern adsorbiertes $\mathrm{CO}_{2}$. Für diese Auffassung können wir folgendes anführen: Titriert man Polyalanin, das aus dem Polymerisationsansatz durch Absaugen des Dioxans und Trocknen i. Vak. über $\mathrm{KOH}$ isoliert wurde, so findet man einen beträchtlichen Verbrauch an Natriummethylat. Löst man das gleiche Polyalanin in Wasser und dampft i. Vak. ein, so findet man nach Trocknen über $\mathrm{KOH}$ uberhaupt keine " $\mathrm{CO}_{2} \mathrm{H}$-Gruppen" mehr. Fällt man das Polyalanin aus seiner wäßrigen Lösung mit Aceton oder Dioxan unter Einleiten von $\mathrm{CO}_{2}$ aus, so wird sogar mehr $\mathrm{CH}_{3} \mathrm{ONa}$ verbraucht als vom ursprünglichen Polyalanin.

Die übrigen Polyaminosäuren sind in Wasser unlöslich, so daß hier analoge Reaktionen nicht durchgeführt werden können. Polyphenylalanin verbraucht regelmäßig besonders viel $\mathrm{CH}_{3} \mathrm{ONa}$. Das gebundene $\mathrm{CO}_{2}$ läßt sich auch durch tagelanges Aufbewahren im Hochvakuum über $\mathrm{KOH}$ nicht entfernen. Wir nehmen an, daß es an die Aminoendgruppen gebunden ist. Zur Bestimmung der Aminosäurekonzentration müssen DCE und $\mathrm{HCl}$ neutrali-

5) R. J. L. Martin, Austral. J. Chem. 10, 268 (1957) [C. A. 52, 5295 (1958)].

6) M. Sela und A. Berger, J. Amer. chem. Soc. 77, 1893 (1955). 
siert werden. Die dabei ausfallenden Peptide nehmen $\mathrm{CO}_{2}$ auf und geben es bei der anschließenden Desaminierung mit Chloramin $\mathrm{T}$ wieder ab. Dadurch wird eine größere Aminosäurekonzentration und ein zu hoher $\mathrm{k}_{\mathrm{E}}$-Wert vorgetäuscht. Da auch relativ niedermolekulare Phenylalanyl-peptide schon unlöslich in Wasser sind, ist hier diese Gefahr besonders groß. Wir haben daher bei der Hydrolyse der Polyphenylalanine Polymerisate mit geringem Polymerisationsgrad als Ausgangsstoffe gewählt.

Der jetzt erhaltene $\mathbf{k}_{\mathrm{E}}$-Wert des Polyphenylalanins liegt zwischen denen des Polynorleucins und Polyleucins, wie es bei einem sterischen Einfluß der Seitenketten auf die Hydrolysengeschwindigkeit erwartet werden muß.

\section{B. Hydrolyse des Dialanyl-15N-alanins}

Bei der Hydrolyse von Poly-DL-alanin in wäßrigem $\mathrm{HCl}$ und in $\mathrm{DCE}-\mathrm{HCl}$ hat sich ergeben ${ }^{2)}$, daß die Geschwindigkeit, mit der endständiges Alanin in Freiheit gesetzt wird, in DCE-HCl fast doppelt so groß ist wie in wäßrigem $\mathrm{HCl}$. Da die gemessene Hydrolysenkonstante $k_{E}=\left(k_{\alpha}+k_{\beta}\right) / 2$ das Mittel aus den Konstanten für die $\mathbf{N}$ - und C-ständige Endbindung ist, könnte 1) $\mathrm{k}_{\alpha}$ und $\mathrm{k}_{\beta}$ beim Wechsel des Reaktionsmediums gleichzeitig erhöht werden; 2) in wäßrigem $\mathrm{HCl}$ eine Peptidkette nur von einem Ende her abgebaut werden, in DCE von beiden Enden her. Wegen der relativ großen Säurestabilität von Dipeptiden ist zu erwarten, daß in wäßrigem $\mathrm{HCl}$ Peptidketten vorwiegend vom Carboxylende her abgebaut werden, denn die $\mathrm{NH}_{3}{ }^{\oplus}$. Endgruppe stößt das angreifende $\mathrm{H}_{3} \mathrm{O}^{\oplus}$ ab.

Um diese Frage zu entscheiden, wurde mit Hilfe der gemischten Anhydridmethode und der Azidmethode ein Dialanyl-alanin synthetisiert, dessen C-endständiges Alanin mit $15 \mathrm{~N}$ markiert war.

Für die Hydrolyse des Tripeptids gelten nachstehende Gleichungen:

$\mathbf{k}_{\boldsymbol{\alpha}}=$ Hydrolysenkonstante der $\mathrm{N}$-endständigen Peptidbindung

$\mathrm{k}_{\beta}=$ Hydrolysenkonstante der C-endständigen Peptidbindung

$\mathrm{k}_{\mathrm{D}}=$ Hydrolysenkonstante des Dipeptids

$\mathbf{T}=$ Konzentration des Tripeptides

D $=$ Konzentration des Dipeptids

A $=$ Konzentration der Aminosäure

Index $0=$ jeweilige Anfangskonzentration

Index $15=15 \mathrm{~N}-$ Gehalt des betreffenden Stoffes

Ohne Berücksichtigung der ${ }^{15} \mathrm{~N}-$ Markierung gilt :

$$
\mathrm{T}+\mathrm{H}_{3} \mathrm{O}^{\oplus} \stackrel{\mathbf{k}_{\alpha}+\mathbf{k}_{\beta}}{\longrightarrow} \mathrm{D}+\mathrm{A} ; \quad \mathrm{D}+\mathrm{H}_{3} \mathrm{O}^{\oplus} \stackrel{\mathbf{k}_{\mathbf{D}}}{\longrightarrow} 2 \mathbf{A}
$$

$-\mathrm{dT} / \mathrm{dt}=\left(\mathrm{k}_{\alpha}+\mathrm{k}_{\beta}\right) \mathrm{T}=2 \mathrm{k}_{\mathrm{E}} \mathrm{T} ; \mathrm{dD} / \mathrm{dt}=2 \mathrm{k}_{\mathrm{E}} \mathrm{T}-\mathrm{k}_{\mathrm{D}} \mathrm{D} ; \mathrm{dA} / \mathrm{dt}=2 \mathrm{k}_{\mathrm{E}} \mathrm{T}+2 \mathrm{k}_{\mathrm{D}} \mathrm{D}$

$3 \mathrm{dT} / \mathrm{dt}+2 \mathrm{dD} / \mathrm{dt}+\mathrm{dA} / \mathrm{dt}=0 ;$ mit $\mathrm{D}_{0}=0$ und $\mathrm{A}_{0}=0$ ist dann

$3 \mathrm{~T}+2 \mathrm{D}+\mathbf{A}=3 \mathrm{~T}_{0}$ oder $\mathbf{A}=3 \mathrm{~T}_{0}-3 \mathrm{~T}-2 \mathrm{D}$, ferner ist $\mathrm{T}=\mathrm{T}_{0} \mathrm{e}^{-2 \mathrm{k}_{\mathrm{E}^{t}}}$

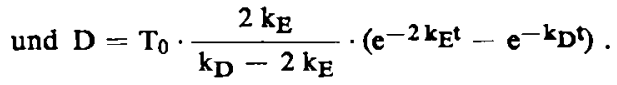


Durch Eirsetzen erhält man dann für die Aminosäurekonzentration A :

$$
A=T_{0}\left[3-\left(3+\frac{4 k_{E}}{k_{D}-2 k_{E}}\right) e^{-2 k_{E}^{t}}+\frac{4 k_{E}}{k_{D}-2 k_{E}} \cdot e^{-k_{D} t}\right]
$$

Beschränkt man sich auf die Bestimmung der Anfangshydrolysengeschwindigkeit und/oder ist $k_{D} \ll k_{E}$, so kann die Hydrolyse der Dipeptide vernachlässigt werden. Die Gleichung (1) geht dann in Gleichung (2) über:

$$
A=T_{0}\left(1-e^{-2 k_{E} t}\right) \text { und } 2.303 \log \frac{T_{0}}{T_{0}-A}=2 k_{E} t
$$

Entsprechend gelten für die Geschwindigkeit der ${ }^{15} \mathrm{~N}$-Alanin-Abspaltung die Gleichungen:

$$
\begin{aligned}
& { }^{15} \mathrm{~T}+\mathrm{H}_{3} \mathrm{O}^{\oplus} \stackrel{\mathrm{k}_{\alpha}}{\longrightarrow}{ }^{15 \mathrm{D}}+\mathrm{A} \quad{ }^{15 \mathrm{~T}}+\mathrm{H}_{3} \mathrm{O}^{\oplus} \stackrel{\mathrm{k}_{\beta}}{\longrightarrow} \mathrm{D}+{ }^{15 \mathrm{~A}} \\
& { }^{15} \mathrm{D}+\mathrm{H}_{3} \mathrm{O}^{\oplus} \stackrel{\mathrm{k}_{\mathrm{D}}}{\longrightarrow} \mathrm{A}+{ }^{15} \mathrm{~A} \quad \mathrm{D}+\mathrm{H}_{3} \mathrm{O}^{\oplus} \stackrel{\mathrm{k}_{\mathrm{D}}}{\longrightarrow} 2 \mathrm{~A}
\end{aligned}
$$

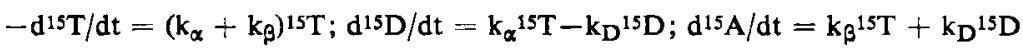

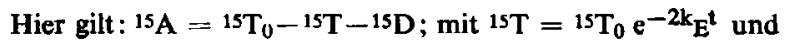

${ }_{15 D}=\frac{k_{\alpha}{ }^{15} T_{0}}{k_{D}-2 k_{E}}\left(e^{-2 k_{E^{t}}}-e^{-k_{D}}\right)$, erhält man fur die Konzentration ${ }^{15} A$ an ${ }^{15} \mathrm{~N}-\mathrm{Alanin}$

Gleichung (3)

$$
{ }^{15} A={ }^{15} T_{0}\left[1-\frac{k_{D}-k_{\beta}}{k_{D}-2 k_{E}} e^{-2 k_{E} t}+\frac{2 k_{E}-k_{\beta}}{k_{D}-2 k_{E}} e^{-k_{D} t}\right]
$$

Um diese Fomeln zu vereinfachen, wird versuchsweise $k_{\alpha} \approx \mathbf{k}_{\mathbf{D}}$ gesetzt. Wenn die Nachbarschaft einer positiven Ladung auf die Spaltung der Peptidbindung einen großen Einfluß hat, ist diese Annahme gerechtfertigt, denn die Dipeptidbindung und die N-endständige Bindung des Tripeptids sind von der $\mathrm{NH}_{3}{ }^{\oplus-G}$-Guppe gleich weit entfernt. Es sind nun zwei Grenzfälle möglich:

a) $k_{\alpha}>k_{\beta}$; das Tripeptid wird vom Aminoende her abgebaut. Die Anfangsgeschwindigkeit, mit der ${ }^{15 \mathrm{~N}}$-Alanin abgespalten wird, ist sehr klein. Je weiter die Hydrolyse fortschreitet, desto größer wird die Bildungsgeschwindigkeit des ${ }^{15} \mathrm{~N}$-Alanins.

b) $k_{\alpha}<k_{\beta}$; das Tripeptid wird vom Carboxylende her abgebaut. In diesem Fall geht für die Anfangsgeschwindigkeit der ${ }^{15} \mathrm{~N}$-Alanin-Bildung Gleichung (3) in Gleichung (4) über:

$$
{ }^{15} \mathrm{~A}={ }^{15} \mathrm{~T}_{0}\left(1-\mathrm{e}^{-\mathrm{k}_{\beta} t}\right) \quad \text { und } \quad 2.303 \log \frac{15 \mathrm{~T}}{15 \mathrm{~T}_{0}-15 \mathrm{~A}}=\mathrm{k}_{\beta} \mathrm{t}
$$

Die Hydrolyse des Dialanyl-15N-alanins wurde einmal durch Bestimmung des insgesamt freiwerdenden Alanins durch Umsetzen mit Chloramin $\mathrm{T}$ verfolgt, zum anderen wurden Proben der Hydrolysenmischung in einer Hochvakuumapparatur mit $\mathrm{HNO}_{2}$ desaminiert und der entstehende Stickstoff massenspektrometrisch auf seinen ${ }^{15} \mathrm{~N}$-Gehalt hin untersucht. 
Den Verlauf der Hydrolyse des 15N-Peptides zeigen die Abbildungen 1 und 2. Die entsprechenden Hydrolysekonstanten sind in Tabelle 2 aufgeführt.

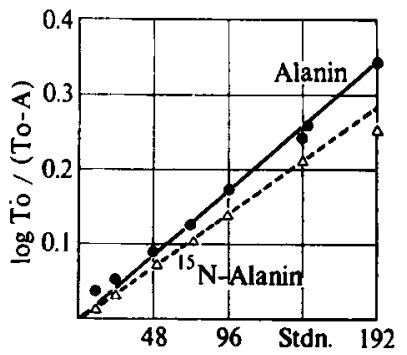

Abbildung 1

Hydrolyse des Dialanyl-15N-alanins bei $37^{\circ}$ in $3.07 n \mathrm{HCl}$

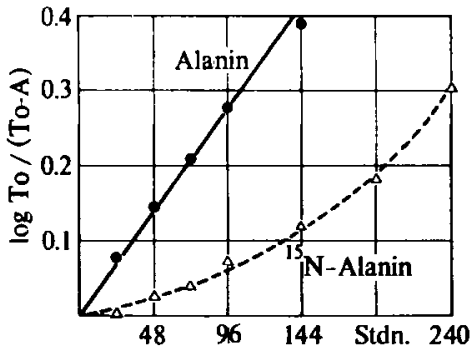

Abbildung 2

Hydrolyse des Dialanyl-15N-alanins bei $37^{\circ}$ in DCE-4.28 $n \mathbf{H C l}$

Tabelle 2. RG-Konstanten der sauren Hydrolyse des Dialanyl-15N-alanins, bezogen auf die analytische $\mathrm{HCl}$-Konzentration $=1 \mathrm{n}$

\begin{tabular}{ccc}
\hline RG-Konstante & in $\mathrm{H}_{2} \mathrm{O}-\mathrm{HCl}$ & in $\mathrm{H}_{2} \mathrm{O}-\mathrm{DCE}-\mathrm{HCl}$ \\
\hline $2 \mathrm{k}_{\mathrm{E}}$ & $38 \cdot 10^{-8}$ Sek. $^{-1}$ & $43 \cdot 10^{-8} \mathrm{Sek}^{-1}$ \\
$\mathrm{k}_{\beta}$ & 30 & $\sim 9$ \\
$\mathrm{k}_{\alpha}$ & 8 & $\sim 34$
\end{tabular}

Zwei Tatsachen fallen auf: 1) Im Gegensatz zum Poly-DL-alanin wird die Gesamtgeschwindigkeit der Hydrolyse des Tripeptids durch DCE nur wenig erhöht. 2) In wäßrigem $\mathrm{HCl}$ wird das Tripeptid bevorzugt an der C-endständigen Bindung gespalten, in DCE-HCl jedoch an der $\mathrm{N}$-endständigen Peptidbindung.

Der bevorzugte Abbau einer Peptidkette am Carboxylende bei der sauren Hydrolyse wurde schon wiederholt angenommen 7). Er ist für Wasser als Lösungsmittel durch diese Versuche endgültig bewiesen. Die Ursache dürfte der elektrostatische Feldeffekt der $\mathbf{N H}_{3}{ }^{\oplus}$-Endgruppe des Peptids sein, der die Ausbildung einer zweiten positiven Ladung (Protonierung) an der benachbarten Peptidbindung verhindert. Der gleiche Effekt kann jedoch nicht die Bevorzugung der $\mathbf{N}$-endständigen Bindung bei der Hydrolyse in 60-70-proz. DCE bewirken, da die herabgesetzte Dielektrizitätskonstante der Mischung die Reichweite eines Feldeffektes verstärken müßte. Wir nehmen an, daß in DCE-HCl die Aminoendgruppe spezifisch durch die Dichloressigsäure solvatisiert wird. Dadurch würde die positive Ladung der $\mathrm{NH}_{3}{ }^{\oplus}$-Gruppe abgeschirmt; daneben wäre es denkbar, daB auf die benachbarte Peptidbindung Protonen direkt von DCE-Molekülen der Solvathülle übertragen werden. Die effektive Protonenkonzentration wäre dann hier höher als in der Lösung. Einen ähnlichen

7) A. H. Gordon, A. J. P. MARTin und R. L. M. SYNGe, Biochem. J. 35, 1369 (1941). 
Effekt diskutieren KerN und SCHERHAG ${ }^{8)}$ bei dem günstigen Einfluß von Polyvinylsulfonsäuren auf die saure Hydrolyse von Peptiden. Eigenartigerweise wird die C-endständige Bindung des Trialanins in der DCE-Lösung kaum angegriffen. Vielleicht wird diese Bindung durch die $\mathrm{CHCl}_{2}$-Gruppen der solvatisierenden DCE-Moleküle für einen hydrolytischen Angriff blockiert. Längere Peptidketten, deren Carboxylende aus dem ,Solvatationsknäuel“ der Aminoendgruppe herausragt, müßten dann an der $\mathrm{C}$-endständigen Bindung normal hydrolysiert werden. Die Verdoppelung von $\mathrm{k}_{\mathrm{E}}$ bei der Hydrolyse des Poly-DL-alanins in DCE-HCl im Vergleich zur Hydrolyse in wäßrigem $\mathrm{HCl}$ stützt diese Vorstellung.

Einige Versuche, die über die alkalische Hydrolyse des Dialanyl-15N-alanins angestellt wurden, zeigen, daß hier die Hydrolyse wesentlich schneller verläuft, $k_{\alpha}, k_{\beta}$ und $k_{D}$ jedoch ungefähr gleich groß sind.

\section{AKTIVIERUNGSENERGIEN UND AKTIVIERUNGSENTROPIEN}

In dem untersuchten Bereich von $25-55^{\circ}$ folgt die Temperaturabhängigkeit der Hydrolysengeschwindigkeit der Polyaminosäuren der ARRHENIUsschen Gleichung $\mathbf{k}=\mathbf{A} \cdot \mathrm{e}^{-\Delta E / R T}$ (Abb. 3). Die Ćbereinstimmung ist in Anbetracht der relativ ungenauen Methode in den meisten Fällen gut. Eine Ausnahme ist Poly-DL-isoleucin, das aber - selbst bei einem mittleren Polymerisationsgrad von nur 10 - in den verwendeten Hydrolysiermischungen nicht vollständig löslich ist, sondern nur stark aufquillt. Die Hydrolyse ist daher auch von der Lösungsgeschwindigkeit abhängig. Die einzelnen ARRHENIUsschen Aktivierungsenergien, Aktionskonstanten, die Aktivierungsenthalpien und -entropien sowie die freien Aktivierungsenthalpien sind in Tabelle 3 angegeben.

Läßt man die Werte für Poly-DL-alanin außer Betracht, so erhält man für die übrigen Polyaminosäuren im Mittel eine Aktivierungsenergie von $24 \mathrm{kcal}$ für eine Endbindung und $23 \mathrm{kcal}$ für eine innere Bindung.

Die Unterschiede in den Hydrolysengeschwindigkeiten dieser Polyaminosäuren werden durch Unterschiede in den Aktionskonstanten bzw. Aktivierungsentropien bestimmt. Die Streuung der einzelnen Aktivierungsenergien $( \pm 1.5 \mathrm{kcal})$ war jedoch so groß, daß ein Gang in den Aktionskonstanten nicht erkennbar war. Diese Konstanten (Tabelle 3) sind daher mit den mittleren Aktivierungsenergien berechnet worden.

Die Aktionskonstanten und Aktivierungsentropien in der Reihe Poly-DL- $\alpha$-aminobuttersäure bis Poly-DL-isoleucin, wie auch die Abstufung der Werte für die Endbindung und die Unterschiede zwischen den Werten für die Endbindungen und inneren Bindungen, sprechen für einen sterischen Effekt der Aminosäureseitenketten. Gleiches gilt für die Werte des Poly-DL-alanins. Die stark negativen Aktivierungsentropien $\Delta \mathbf{S} \neq$,

8) W. Kern und B. Scherhag, Makromolekulare Chem. 28, 209 (1958). 


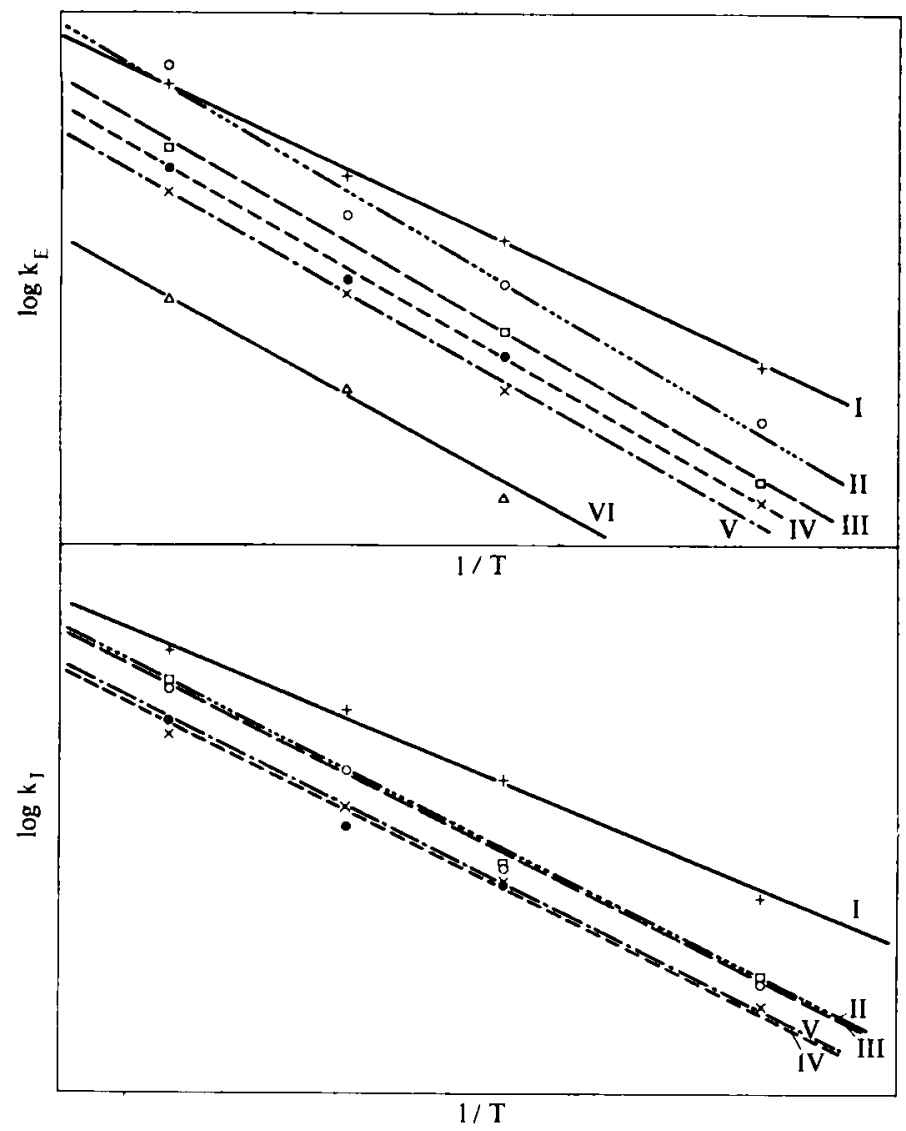

Abbildung 3. Temperaturabhängigkeit der Hydrolyse der Poly- $\alpha$-aminosäuren
1 - $-\quad$ Poly- $\alpha$-alanin
II ..-०-.- Poly- $\alpha$-amino-buttersäure
IV -.-๑-- Poly-phenylalanin
III - $\square-ー$ Poly-norleucin
V - $\times$ - Poly-leucin
VI $-\Delta-$ Poly-isoleucin

Tabelle 3. Konstanten der sauren Peptidhydrolyse bei $37^{\circ}$

\begin{tabular}{|c|c|c|c|c|c|c|c|c|c|c|}
\hline \multirow{2}{*}{$\begin{array}{l}\text { Poly- } \\
\text { aminosäure }\end{array}$} & \multicolumn{5}{|c|}{ für die Endbindung } & \multicolumn{5}{|c|}{ für eine innere Bindung } \\
\hline & $\Delta \mathrm{E}$ & $\mathbf{A}$ & $\Delta H^{\neq}$ & $\Delta \mathbf{S}^{\neq}$ & $\Delta \mathbf{G}^{\neq}$ & $\Delta \mathrm{E}$ & $\mathbf{A}$ & $\Delta \mathbf{H}^{\neq} \neq$ & $\Delta \mathbf{S} \neq$ & $\Delta \mathrm{G}^{\neq}$ \\
\hline Poly & 21 & $7.7 \cdot 10^{8}$ & 20.7 & -20 & 27 & 20 & $2.3 \cdot 10^{7}$ & 19.2 & -27 & 28 \\
\hline Poly-DL- $\alpha$-amb & 23 & $5.8 \cdot 10^{10 * 1}$ & 22.6 & -15 & 27 & 22 & $1.3 \cdot 10^{9 *)}$ & 21.6 & -21 & 28 \\
\hline Poly-DL-norleu & 25 & $3.2 \cdot 10^{10} * 1$ & 24.8 & -9 & 28 & 23 & $\left.1.1 \cdot 10^{9} *\right)$ & 22.4 & -18 & 28 \\
\hline Poly-DL-phe & 25 & $\left.2.4 \cdot 10^{10} \neq\right)$ & 23.9 & -12 & 29 & 24 & $0.8 \cdot 10^{9 * 1}$ & 23.4 & -16 & 28 \\
\hline Poly-DL-leu & 24 & $2.0 \cdot 10^{10}$ *) & 23.3 & -15 & 28 & 22 & $\left.0.8 \cdot 10^{9} *\right)$ & 21.4 & -22 & 28 \\
\hline Poly-DL-isoleu & 24 & $0.5 \cdot 1010 *)$ & 23.4 & -17 & 29 & 一 & - & - & - & - \\
\hline
\end{tabular}

*) berechnet aus der mittleren Aktivierungsenergie $24 \mathrm{kcal}$ bzw. $23 \mathrm{kcal}$ 
$-20 \mathrm{cal} / \mathrm{Grad}$ für die Endbindung und $-27 \mathrm{cal} / \mathrm{Grad}$ für die innere Bindung, zeigen eine geringe Bildungswahrscheinlichkeit des Übergangszustandes der Hydrolyse an. Kalottenmodelle zeigen, daß die Methylseitenkette im Polyalanin die Peptidbindung relativ wenig sterisch hindert. Infolgedessen steigt hier die sterische Hinderung beim Übergang zum tetraedrischen Übergangszustand stärker an als bei den anderen Polyaminosäuren, da schon die Äthylseitenkette in der Poly-DL- $\alpha$-amino-buttersäure die Peptidbindung auch im Ausgangszustand stark behindert. Da nach diesen Vorstellungen Freiheitsgrade der inneren Rotation und Schwingung im Übergangszustand des Poly-DL-alanins „einfrieren“, wodurch der Beitrag an kinetischer Energie zur Aktivierungsenergie herabgesetzt wird, ist auch die um jeweils 3 kcal kleinere Aktivierungsenergie der Hydrolyse des Poly-DL-alanins kein Beweis für das Auftreten induktiver Effekte bei der sauren Peptidhydrolyse.

\section{BESCHREIBUNG DER VERSUCHE}

\section{Die Polypeptide}

Die Darstellung von Poly-DL-alanin, Poly-DL- $\alpha$-amino-buttersäure, Poly-DL-norleucin, Poly-DL-phenylalanin, Poly-DL-leucin und Poly-DL-isoleucin wurde bereits beschrieben ${ }^{11}$.

\section{Poly-DL-[p-methoxy-phenyl $]$-alanin}

a) 4-[p-Methoxy-benzyl]-DL-oxazoliddion-(2.5). - $9.5 \mathrm{~g}$ DL-[p-Methoxy-phenyl]-alanin wurden in $250 \mathrm{ccm}$ Dioxan suspendiert und bei ca. $45^{\circ}$ mit Phosgen behandelt. Nach 50 bis 60 Min. war die Aminosäure in Lösung gegangen. Nach Abdestillieren des Dioxans i. Vak. kristallisierte der Rückstand sofort. Aus Chloroform sechseckige oder rhombische Blättchen, Schmp. $146-148^{\circ}$ (unkorr.). Ausbeute $10.3 \mathrm{~g}$ (95\% d. Th.).

b) Poly-DL-[p-methoxy-phenyl]-alanin. $-9.5 \mathrm{~g}$ des Oxazoliddions- (2.5) wurden in $190 \mathrm{ccm}$ Dioxan gelöst und nach Zusatz von $9.5 \mathrm{ccm}$ Wasser auf dem Wasserbad erhitzt. Nach ca. 30 Min. trübte sich die Lösung, und das Polypeptid flockte aus. Ausbeute, nach Auskochen mit Wasser und Trocknen über $\mathrm{KOH}$ und $\mathrm{H}_{2} \mathrm{SO}_{4}, 7.3 \mathrm{~g}(93 \% \mathrm{~d}$. Th.). In der Polyaminosäure konnten chromatographisch keine mit Ninhydrin anfärbbaren Peptide nachgewiesen werden. Mittlerer Polymerisationsgrad 19.9.

\section{Poly-DL-[p-nitro-phenyl]-alanin}

a) 4-[p-Nitro-benzyl]-DL-oxazoliddion-(2.5). - $10.6 \mathrm{~g}$ DL-[p-Nitro-phenyl]-alanin hat man in $250 \mathrm{ccm}$ Dioxan suspendiert und bei $45^{\circ}$ Phosgen eingeleitet. Nach 330 Min. war die Lösung klar. Das Dioxan wurde i. Vak. abgedampft und der kristalline Rückstand zweimal aus Dioxan/Petroläther umkristallisiert. Schwach gelbliche, derbe Nadeln, die beim langsamen Erhitzen ohne zu schmelzen polymerisieren. Beim schnellen Erhitzen Schmp. 182 (unkorr.). Ausbeute $9.8 \mathrm{~g}(83 \%$ d. Th. $)$.

b) Poly-DL-[p-nitro-phenyl]-alanin. $-9.2 \mathrm{~g}$ des Oxazoliddions- $(2.5)$ wurden in $185 \mathrm{ccm}$ Dioxan gelöst und $1.9 \mathrm{ccm}$ Wasser zugesetzt. Beim Erwärmen trat schnell eine leichte Trübung auf, die sich jedoch auch bei längerem Kochen nicht verstärkte. Erst nach Erhöhung des 
Wassergehaltes auf $3 \%$ und $5 \mathrm{stdg}$. Kochen auf dem Wasserbad flockte die Polyaminosäure aus. Nach Abfiltrieren und Waschen mit Dioxan und heißem Wasser wurden $3.2 \mathrm{~g}(43 \%$ d. Th.) eines ockergelben Pulvers erhalten. Das Chromatogramm in Butanol/Eisessig zeigte keine mit Ninhydrin anfärbbaren Anteile. Das Polymerisat ist ebenso wie p-Nitro-phenylalanin gegen Alkalien empfindlich.

\section{Synthese des Dialanyl-15N-alanins}

${ }^{15 N} \mathrm{~N}$-Alanin wurde aus Phthalimid-15N und $\alpha$-Brom-propionsäureäthylester dargestelit 9 ). Der ${ }^{15 N}$-Gehalt des Alanins betrug 9.47\%. Carbobenzoxyalanylalaninmethylester wurde nach der gemischten Anhydridmethode durch Kuppeln der beiden Komponenten mittels Chlorameisensäureäthylesters dargestellt und nach bekannter Vorschrift 10) in das Azid ubergeführt.

Eine Lösung von $3.9 \mathrm{~g}$ frisch hergestelltem Carbobenzoxyalanylalaninazid in Äther wird zu einer ätherischen Lösung von $0.02 \mathrm{Mol}{ }^{15} \mathrm{~N}$-Alaninbenzylester gegeben. Uber Nacht scheidet sich der Carbobenzoxy-dialanyl-15 $\mathrm{N}$-alaninbenzylester kristallin ab. Die Kristalle werden abgesaugt, in Essigester aufgenommen und die Lösung mit verd. Salzsäure, verd. $\mathrm{NaHCO}_{3}-$ Lösung und zum Schluß mit Wasser durchgeschüttelt. Nach dem Trocknen über $\mathrm{Na}_{2} \mathrm{SO}_{4}$ wird der Essigester abdestilliert. Ausbeute an Carbobenzoxytripeptid-benzylester $5.5 \mathrm{~g}$ (61\% d. Th.). Der Ester wird in $200 \mathrm{ccm}$ absol. Methanol gelöst, mit $10 \mathrm{ccm}$ Eisessig und $\boldsymbol{P d}$-Katalysator (aus $\mathbf{2} \mathrm{g} \mathrm{PdCl}_{2}$ ) versetzt und die Benzylgruppe und der Carbobenzoxyrest bei $60^{\circ}$ abhydriert. Das Tripeptid fällt aus der methanolischen Lösung aus. Ausbeute $2.8 \mathrm{~g}$. Der ${ }^{15} \mathrm{~N}-$ Gehalt wurde nach Totalhydrolyse mit $6 n \mathrm{HCl}$ bei $110^{\circ} \mathrm{zu} 9.51 \%$ bestimmt.

\section{Hydrolyse der Polypeptide}

Als Beispiel für die Hydrolyse der Polyaminosäuren sei in Tabelle 4 der Hydrolysenverlauf des Poly-DL-alanins bei $37^{\circ}$ angegeben.

Tabelle 4. Hydrolyse von Poly-DL-alanin

\begin{tabular}{rlll}
$\begin{array}{c}\mathrm{t} \\
\text { (Stdn.) }\end{array}$ & $\begin{array}{c}{[\mathrm{A}]} \\
(\mathrm{Mol} / l)\end{array}$ & $\begin{array}{c}{\left[\mathrm{NH}_{2}\right]_{\mathrm{t}}} \\
(\mathrm{Mol} / l)\end{array}$ & $\begin{array}{c}{\left[\mathrm{NH}_{2}\right]_{\mathrm{t}}-[\mathrm{A}]} \\
(\mathrm{Mol} / l)\end{array}$ \\
\hline 3 & $0.13 \cdot 10^{-2}$ & - & - \\
6 & 0.26 & $2.82 \cdot 10^{-2}$ & $2.56 \cdot 10^{-2}$ \\
12 & 0.38 & 4.25 & 3.87 \\
18 & 0.58 & 6.87 & 6.29 \\
24 & 0.81 & 6.47 & 5.66 \\
32 & 1.25 & 8.52 & 7.27 \\
38 & 1.44 & 9.67 & 8.23 \\
48 & 1.87 & 11.22 & 9.35
\end{tabular}

Poly-DL-alanin: mittlerer Polymerisationsgrad 53.9. $\mathrm{HCl}-$ Konzentration: $\left[\mathrm{H}_{3} \mathrm{O}^{\oplus}\right]=4.85 \mathrm{n}$. Konzentration der gesamten Alaninreste $[A R]=50.0 \cdot 10^{-2} \mathrm{Mol} / l$. Anfangskonzentration der Aminoendgruppen : $\left[\mathrm{NH}_{2}\right]_{\mathrm{t}=0}=0.93 \cdot 10^{-2} \mathrm{Mol} / l$. Anfangskonzentration des Alanins:

$$
[A]_{t=0}=0
$$

9) vgl. R. Schoenheimer und S. RATner, J. biol. Chemistry 127, 301 (1939).

10) M. Bergmann und L. Zervas, J. biol. Chemistry 113, 341 (1936). 
Aus den Kurven für [A] und ([NH $]_{t}-[\mathrm{A}]$ ) (Abb. 4 und 5) wurden nach dem bereits in den vorhergehenden Mitteilungen beschriebenen Verfahren graphisch $k_{\mathbf{E}} z \mathrm{z} 83.3 \cdot 10^{-8}$ Sek.-1 und kI zu $27.3 \cdot 10^{-8}$ Sek. $^{-1}$ ermittelt.
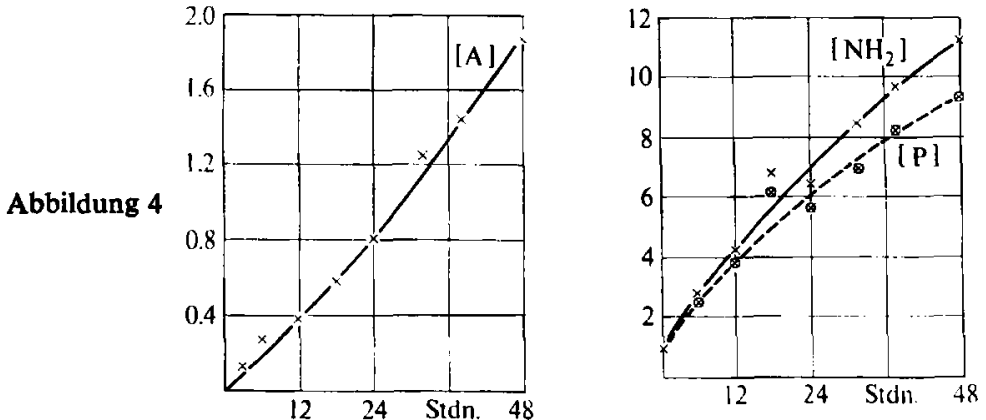

Abbildung 5

Hydrolyse des Poly-DL-alanins bei $37^{\circ}$

Abb. 4. Ordinaten: [A] in $10^{-2} \mathrm{Mol} / l$; Abb. 5. Ordinaten: $\left[\mathrm{NH}_{2}\right]$ und $\left[\mathrm{NH}_{2}\right]_{\mathfrak{t}}-[\mathrm{A}]=[\mathrm{P}]$

(Konzentration an polymerem Alanin) in $10^{-2} \mathrm{Mol} / \mathrm{l}$

Die auf gleiche Weise bestimmten Hydrolysekonstanten der Polyaminosäuren sind in Tabelle 5 zusammengefaßt*).

Tabelle S. Hydrolysekonstanten

\begin{tabular}{|c|c|c|c|c|c|c|c|c|}
\hline \multirow{2}{*}{$\begin{array}{c}\text { Poly- } \\
\text { aminosäure }\end{array}$} & \multicolumn{4}{|c|}{$\mathrm{k}_{\mathrm{E}} \cdot 10^{8}$ Sek..$^{-1}$} & \multicolumn{4}{|c|}{$\mathrm{k}_{\mathrm{I}} \cdot 10^{8}$ Sek. ${ }^{-1}$} \\
\hline & $25^{\circ}$ & $37^{\circ}$ & $45^{\circ}$ & $54.4^{\circ}$ & $25^{\circ}$ & $37^{\circ}$ & $45^{\circ}$ & $54.4^{\circ}$ \\
\hline Poly-DL-ala & 19.2 & 83.3 & 173.0 & 486 & 7.0 & 27.3 & 62.3 & 130 \\
\hline Poly-DL- $\alpha$-amb & 10.1 & 49.5 & 107.6 & 498 & 2.7 & 10.8 & 32.0 & 81.9 \\
\hline Poly-DL-norleu & 5.2 & 27.2 & - & 238 & 2.7 & 9.9 & - & 88.7 \\
\hline Poly-DL-leu & 4.3 & 14.0 & 43.6 & 138 & 2.0 & 8.3 & 22.5 & 47.3 \\
\hline Poly-DL-phe & - & 21.3 & 50.7 & 180 & - & 7.6 & 15.6 & 60.9 \\
\hline Poly-DL-isoleu & - & 4.1 & 14.7 & 40.6 & - & 2.5 & 5.3 & 9.8 \\
\hline
\end{tabular}

Die ArRhenrusschen Aktivierungsenergien wurden aus einem Diagramm log (k) gegen 1/ $\mathrm{T}$ graphisch bestimmt. Die Aktivierungsenthalpien wurden nach $\Delta H \neq=\Delta \mathrm{E}-\mathrm{RT}$ berechnet, die freien Aktivierungsenthalpien und die Aktivierungsentropien mit Hilfe der Gleichung

$$
k_{E} \text { bzw. } k_{I}=(k T / h) \cdot e^{\Delta S \neq / R} \cdot e^{-\Delta H} \neq / R T=(k T / h) \cdot e^{-\Delta G \neq / R T}
$$

Hydrolyse des Dialanyl-15N-alanins: $2.5 \mathrm{mMol}$ des Tripeptids wurden in $50 \mathrm{ccm}$ der jeweiligen Hydrolysenmischung gelöst und in einem Meßkolben in einen Thermostat von $37^{\circ}$ getaucht. Der Mischung wurden in bestimmten Zeitabständen $1 \mathrm{ccm}$ zur Aminosäurebestimmung nach der Chloramin-T-Methode und $4 \mathrm{ccm}$ zur Bestimmung der ${ }^{15 \mathrm{~N}}$-Konzentration entnommen. Zur ${ }^{15 N}$-Bestimmung wurden die $4 \mathrm{ccm}$ mit $\mathrm{NaOH}$ neutralisiert, mit

*) Die einzelnen Meßwerte sind in der Dissertation H. F. GrürzMacher, Univ. Hamburg 1959 , angegeben. 
$4 \mathrm{ccm}$ Eisessig-Acetat-Puffer versetzt und in ein Zersetzungskölbchen gegeben, das in einem schwenkbaren seitlichen Ansatz $2 \mathrm{ccm}$ 30-proz. $\mathrm{NaNO}_{2}$-Lösung enthielt. Nach Anschließen des Kölbchens an eine Hochvakuumapparatur und Evakuieren wurde durch Schwenken des seitlichen Ansatzes die $\mathrm{NaNO}_{2}$-Lösung mit der Aminosäurelösung gemischt. Nach halbstündiger Reaktion wurde das reichlich entstandene NO mit flüssiger Luft ausgefroren und das restliche Gas mit Hilfe einer TöPlER-Pumpe in ein Probengefäß übergeführt. Der ${ }^{15 N-}$ Gehalt des Gases wurde mit einem $\mathrm{CH}_{4}$-Massenspektrometer der ATLAS-Werke, Bremen, bestimmt. Durch Kombination eines Massenprogrammwählers mit einer Kompensationsschaltung konnte direkt das Verhältnis der Intensitäten der Ionenströme der Massen 29 und 28 abgelesen werden. Bezeichnet man dieses Verhältnis $I_{29} / \mathbf{l}_{28}$ als $\chi$, so ist der Prozentgehalt an ${ }^{15} \mathrm{~N}$ in der Probe: $\%{ }^{15} \mathrm{~N}=100 \chi /(2+\chi)$.

Im Anfangsstadium der Hydrolyse ist die Gesamtkonzentration der Aminogruppen gleich der Anfangskonzentration des Tripeptids $\left(T_{0}\right)$ und der Konzentration der Aminosäuren (A). Mit Hilfe des ${ }^{15} \mathrm{~N}$-Prozentgehaltes der C-endständigen Peptidbindung von 9.515 und des ${ }_{15} \mathrm{~N}$-Prozentgehaltes des normalen Stickstoffes von 0.365 erhält man so für die Konzentration der vom Carboxylende des Tripeptids stammenden Aminosäure

$$
15 \mathrm{~A}=\left(\mathrm{T}_{0}+\mathrm{A}\right) \frac{2(\% 15 \mathrm{~N}-0.365)}{9.515-0.365}
$$

Die Meßwerte für die Hydrolyse des Dialanyl-15N-alanins in $3.07 n \mathrm{HCl}$ und in DCE$4.28 n \mathrm{HCl}$ sind in den Tabellen 6 und 7 aufgeführt:

Tabelle 6. Hydrolyse in $3.07 n \mathrm{HCl}$ bei $37^{\circ}$

\begin{tabular}{rcccc} 
& $\mathrm{T}_{0}=0.047 \mathrm{Mol} / l$, & $\mathrm{A}_{0}=0.010 \mathrm{Mol} / l$, & \multicolumn{2}{c}{$15 \mathrm{~A}_{0}=0.0035 \mathrm{Mol} / l$} \\
\hline $\begin{array}{c}\mathrm{t} \\
\text { (Stdn.) }\end{array}$ & $\mathrm{A}$ & $\mathrm{T}_{0}-\mathrm{A}$ & $\log \left(\mathrm{T}_{0} / \mathrm{T}_{0}-\mathrm{A}\right)$ & $1 / \mathrm{t} \cdot \log \left(\mathrm{T}_{0} / \mathrm{T}_{\mathbf{0}}-\mathrm{A}\right)$ \\
\hline 6 & 0.0103 & 0.0463 & 0.0065 & $(0.0011)$ \\
12 & 0.0140 & 0.0430 & 0.0386 & $(0.0032)$ \\
24 & 0.0153 & 0.0417 & 0.0520 & 0.0021 \\
48 & 0.0189 & 0.0381 & 0.0912 & 0.0019 \\
72 & 0.0221 & 0.0349 & 0.1293 & 0.0018 \\
96 & 0.0256 & 0.0314 & 0.1752 & 0.0018 \\
144 & 0.0302 & 0.0268 & 0.2440 & 0.0017 \\
148 & 0.0312 & 0.0258 & 0.2605 & 0.0018 \\
192 & 0.0358 & 0.0212 & 0.3458 & 0.0018
\end{tabular}

\begin{tabular}{rccccc}
\hline $\begin{array}{c}t \\
\text { (Stdn.) }\end{array}$ & $\%{ }^{15 N}$ & $15 \mathrm{~A}$ & $\mathrm{~T}_{0}-{ }^{15} \mathrm{~A}$ & $\log \left(\mathrm{T}_{0} / \mathrm{T}_{0}-{ }^{15} \mathrm{~A}\right)$ & $1 / \mathrm{t} \cdot \log \left(\mathrm{T}_{0} / \mathrm{T}_{0}-{ }^{15} \mathrm{~A}\right)$ \\
\hline 12 & 0.761 & 0.0053 & 0.0452 & 0.0170 & 0.0014 \\
24 & 0.874 & 0.0070 & 0.0435 & 0.0336 & 0.0014 \\
50 & 1.124 & 0.0110 & 0.0395 & 0.0755 & 0.0015 \\
74 & 1.249 & 0.0134 & 0.0371 & 0.1027 & 0.0014 \\
96 & 1.399 & 0.0164 & 0.0341 & 0.1393 & 0.0014 \\
144 & 1.685 & 0.0216 & 0.0289 & 0.2112 & 0.0015 \\
192 & 1.701 & 0.0243 & 0.0262 & 0.2538 & 0.0013
\end{tabular}


Tabelle 7. Hydrolyse in DCE-HCl-Mischung $(4.28 n \mathrm{HCl})$

$\mathrm{T}_{0}=0.0470 \mathrm{Mol} / l, \quad \mathrm{~A}_{0}=0.010 \mathrm{Mol} / l, \quad{ }^{15} \mathrm{~A}_{0}=0.0061 \mathrm{Mol} / l$

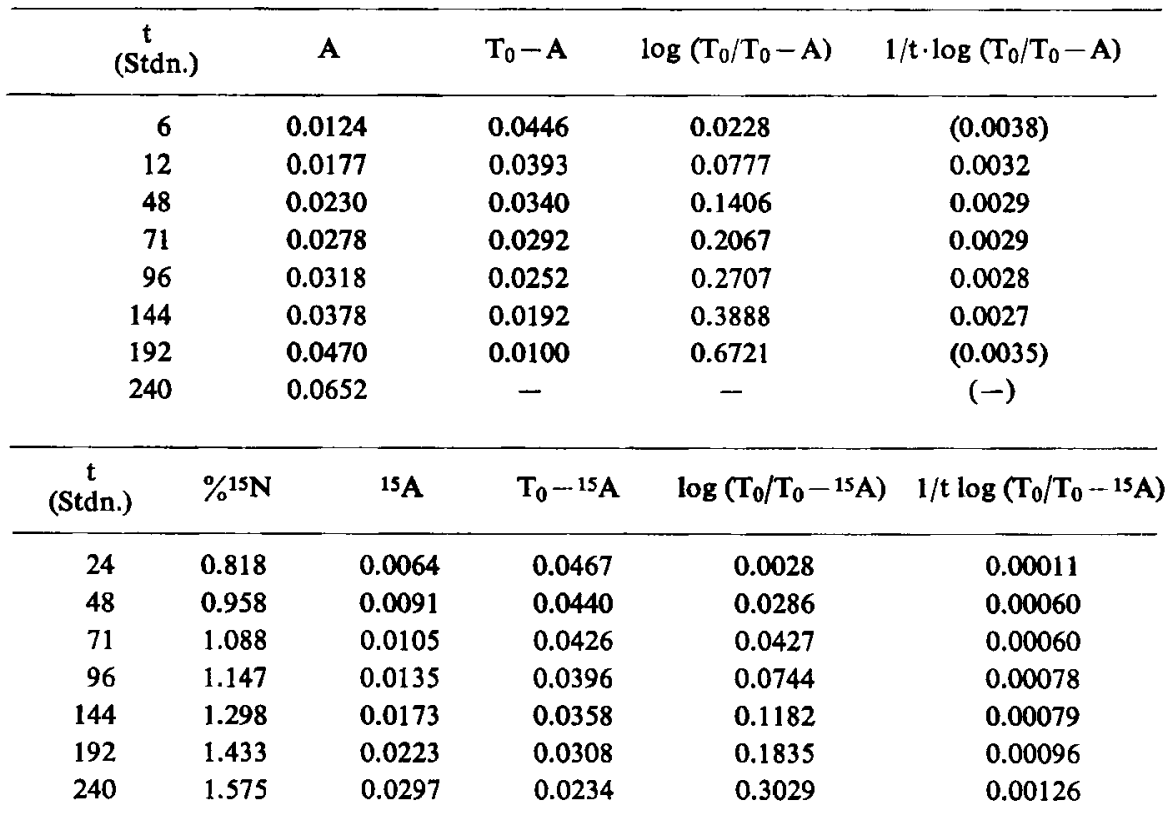

